\title{
Spectrally Selective Ultrathin Photodetectors Using Strong Interference in Nanocavity Design
}

\author{
Amir Ghobadi ${ }^{\circledR}$, Yigit Demirag, Hodjat Hajian, Ahmet Toprak, Bayram Butun ${ }^{\circledR}$, and Ekmel Ozbay
}

\begin{abstract}
Thinning the active layer's thickness of the semiconductor down to a level comparable with the carriers' diffusion length while keeping its absorption high is an ultimate goal to boost the performance of optoelectronic devices. Strong interference in multilayer structures is one of the promising and practical solutions owing to their simple and large-scale compatible fabrication route. These nanocavity designs not only provide near unity absorption, but they can also be designed in a way that a spectrally selective absorption response can be achieved. In this letter, we will demonstrate the functionality of a metalinsulator-semiconductor (MIS) cavity to obtain spectrally selective ultrathin photodetectors. To prove our theoretical and numerical findings, a 4-nm-thick amorphous silicon (aSi)-based MIS cavity is designed, fabricated, and characterized. The experimental results show that the optimized cavity design can act as an efficient visible blind ultraviolet (UV) photodetector. The proposed design shows the responsivity values of 120 and $2.5 \mathrm{~mA} / W$ in the UV $(\lambda=350 \mathrm{~nm})$ and visible $(\lambda=500 \mathrm{~nm})$ regions, respectively.
\end{abstract}

Index Terms-Perfect absorbers, optical devices, semiconductor metamaterials, photodetectors.

\section{INTRODUCTION}

$\mathbf{P}$ ERFECT light absorbers (PLAs) have been the subject of intensive studies in recent years. Owing to their relatively large extinction coefficient in the broad range of the electromagnetic (EM) spectrum, the commonly utilized material in these PLAs are sub-wavelength metal nanostructures. The use of plasmonic nanopatterns in single or multilayer cavity structures have been the main strategy for the design of PLAs [1]-[6]. However, electron beam lithography (EBL) is

Manuscript received February 21, 2019; revised March 27, 2019; accepted April 4, 2019. Date of publication April 11, 2019; date of current version May 23, 2019. The review of this letter was arranged by Editor S. J. Koester. (Corresponding author: Amir Ghobadi.)

A. Ghobadi is with the Department of Electrical and Electronics Engineering, Bilkent University, 06800 Ankara, Turkey, and also with the Nanotechnology Research Center, Bilkent University, 06800 Ankara, Turkey (e-mail: amir@ee.bilkent.edu.tr).

Y. Demirag, H. Hajian, A. Toprak, and B. Butun are with the Nanotechnology Research Center, Bilkent University, 06800 Ankara, Turkey.

E. Ozbay is with the Nanotechnology Research Center, Bilkent University, 06800 Ankara, Turkey, also with the Department of Electrical and Electronics Engineering, Institute of Materials Science and Nanotechnology, UNAM, Bilkent University, 06800 Ankara, Turkey and also with the Department of Physics, Bilkent University, 06800 Ankara, Turkey.

Color versions of one or more of the figures in this letter are available online at http://ieeexplore.ieee.org.

Digital Object Identifier 10.1109/LED.2019.2910064 involved in the fabrication of these subwavelength nano units which further limits their upscaling for practical applications. Therefore, it is highly desired to design a planar multilayer based PLA architecture that can retain the impedance matching condition in the intended wavelength regime. Recently, many EBL-free metal based PLA designs have been proposed for this purpose [7]-[14].

These PLAs can also be realized in planar semiconductor based metasurfaces and they can go beyond the Yablonovitch limit [15]-[21]. These metal-semiconductor (MS) designs could be of particular interest in the vast variety of optoelectronic applications. In a recent Perspective [22], we showed that strong light absorption can be realized using lithography-free planar semiconductor based metamaterials, in a broad or narrow frequency range, upon the proper choice of material and configuration. Our theoretical findings reveal that the use of a metal-insulator-semiconductor (MIS) configuration (instead of MS design) adds a new degree of freedom to improve the design response. By tuning the spacer layer thickness, it is possible to tune the operation wavelength [23] and bandwidth (BW) of the design [24]. Therefore, PLA can be acquired in dimensions much smaller than the carriers' diffusion length. Considering the fact that semiconductors in such thin thicknesses are amorphous and have a short diffusion length [25], the total absorption of light in nanometer scale dimensions can significantly increase the carriers' collection efficiency. Therefore, the concept of photodetectors with cavity enhancement have been the subject of several studies [23], [26]-[28].

In this letter, a spectrally selective visible blind ultraviolet (UV) photodetector is fabricated using a $4 \mathrm{~nm}$ thick a-Si layer in an MIS architecture. The proposed cavity design is designed in a way that the ultrathin semiconductor layer undergoes a strong light-matter interaction in the UV range while it is transparent into the incident visible light. The resonant mode in the cavity is adjusted with both spacer and top layer thicknesses to optimize the detector UV response. The optimized design has above 70 percent absorption in UV and only 2 percent in the visible range. This leads to an approx. two orders of magnitude difference in the photoresponsivity of the design between these two spectral regions. The design shows responsivity values of $120 \mathrm{~mA} / \mathrm{W}$ and $2.5 \mathrm{~mA} / \mathrm{W}$ in the UV $(\lambda=350 \mathrm{~nm})$ and visible $(\lambda=500 \mathrm{~nm})$ regions, respectively. 


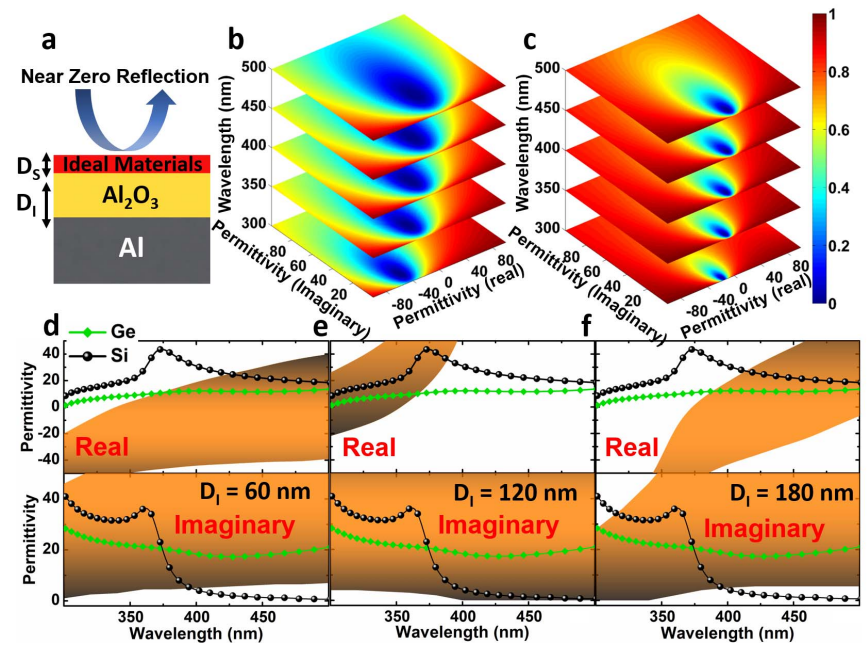

Fig. 1. (a) The schematic representation of MIS cavity design and its geometries. (b) The reflection contour plots as a function of real and imaginary parts of permittivity in different wavelength values for (b) $D_{S}=$ $3 \mathrm{~nm}$, (c) $D_{S}=10 \mathrm{~nm}$. The insulator is fixed at $60 \mathrm{~nm}$. The extracted ideal regions and their matching with the permittivity data of $\mathrm{Si}$ and $\mathrm{Ge}$

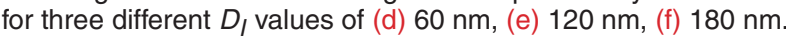

It is envisioned that the use of other semiconductors in proper cavity geometries could lead to spectrally selective photodetectors.

\section{Results and Discussion}

To begin with, a theoretical method based on the transfer matrix method (TMM) is adopted to find the ideal permittivity data for light perfect absorption in a MIS configuration [22], as depicted in Fig. 1(a). In this model, the bottom layer is thick aluminium ( $\mathrm{Al}$ ) mirror, the spacer is aluminum oxide $\left(\mathrm{Al}_{2} \mathrm{O}_{3}\right)$ with a thickness of $\mathrm{D}_{\mathrm{I}}$. The top ideal material has been extracted for a $\mathrm{D}_{\mathrm{S}}$ thick layer. The reflection contour plots at different wavelengths have been extracted for two different $D_{M}$ thicknesses of $3 \mathrm{~nm}$, and $10 \mathrm{~nm}$, as shown in Fig. 1(b)-(c). As these contour plots show, the reflection near zero regions (the bluish circles) are much wider for the thinner top layer. Thus, the probability that a semiconductor permittivity locates inside the ideal perfect absorption region is higher for thinner top layers. Therefore, the $D_{S}$ is fixed at $3 \mathrm{~nm}$ and this time the impact of $\mathrm{D}_{\mathrm{I}}$ is investigated. For this aim, the tolerable real $\left(\varepsilon_{\text {real }}\right)$ and imaginary $\left(\varepsilon_{\text {imaginary }}\right)$ parts of permittivity for a reflection below 0.2 (corresponding to an absorption above 0.8 ) is extracted for $D_{I}=60 \mathrm{~nm}$, $120 \mathrm{~nm}$, and $180 \mathrm{~nm}$, as shown in Fig. 1(d)-(f). In other words, the highlighted region shows the borders of blue circles for $\varepsilon_{\text {real }}$ and $\varepsilon_{\text {imaginary }}$ where the reflection is below 0.2 . These ideal regions are compared with the permittivity data for $\mathrm{Si}$ and Germanium $(\mathrm{Ge})$. For the case of $\mathrm{Si}$, the matching of $\varepsilon_{\text {imaginary }}$ is only met for the shorter wavelengths while the real part matching is tuned as we change the $\mathrm{D}_{\mathrm{I}}$. At the specific dimension of $\mathrm{D}_{\mathrm{I}}=120 \mathrm{~nm}$, we have matching in both parts. However, for Ge, the imaginary part matching is obtained in the whole range and the matching of real part experiences a red shift as we go to thicker spacer layers (see Fig. 1(e)-(f)). Therefore, while $\mathrm{Si}$ acts as an UV absorber, Ge can reveal
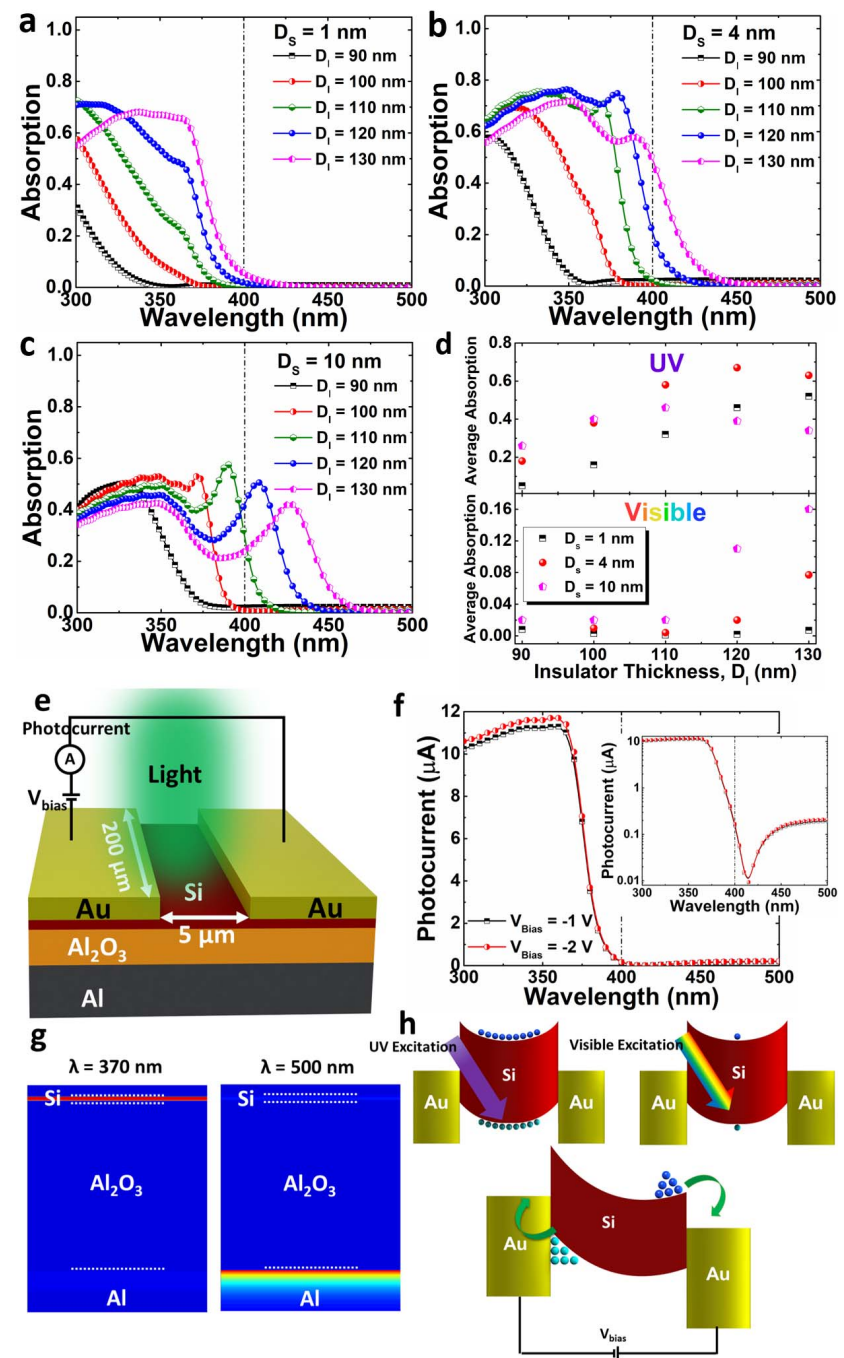

Fig. 2. The absorbed power within Si slab for different $D_{S}$ values of (a) $1 \mathrm{~nm}$, (b) $4 \mathrm{~nm}$, and (c) $10 \mathrm{~nm}$. (d) Average light absorption in UV and visible regimes for different design geometries. (e) The proposed MSM photodetector made on MIS cavity, (f) simulated photocurrent values of the design as a function of light wavelength, ( $g$ ) light absorption across the cavity in two wavelengths of $\lambda=370 \mathrm{~nm}$, and $500 \mathrm{~nm}$, and (h) representation of device operation under UV and visible light irradiation.

spectrally selective visible perfect absorption by tuning the $\mathrm{D}_{\mathrm{I}}$, which is in line with previous studies [23].

Taking this theoretical estimations into account, numerical simulations, using a commercial finite-difference timedomain (FDTD) software package [29], are further employed to investigate the absorption capability of the MIS design. Based on our theoretical calculations, we will focus on the design of a silicon metasurface based visible blind UV photodetector. To further optimize our MIS design, we need to find the amount of the absorbed power inside the ultrathin $\mathrm{Si}$ coating to maximize the UV absorption while minimizing the visible response. For this, following formula is employed:

$$
A(\lambda)=\frac{1}{P_{\text {incident }}} 0.5 \omega \operatorname{imag}\left(\varepsilon_{S i}\right)|E|^{2}
$$

where $\mathrm{E}$ is the electric field inside the $\mathrm{Si}$ layer. For this purpose, three $D_{S}$ values of $1 \mathrm{~nm}, 4 \mathrm{~nm}$, and $10 \mathrm{~nm}$ are selected. 

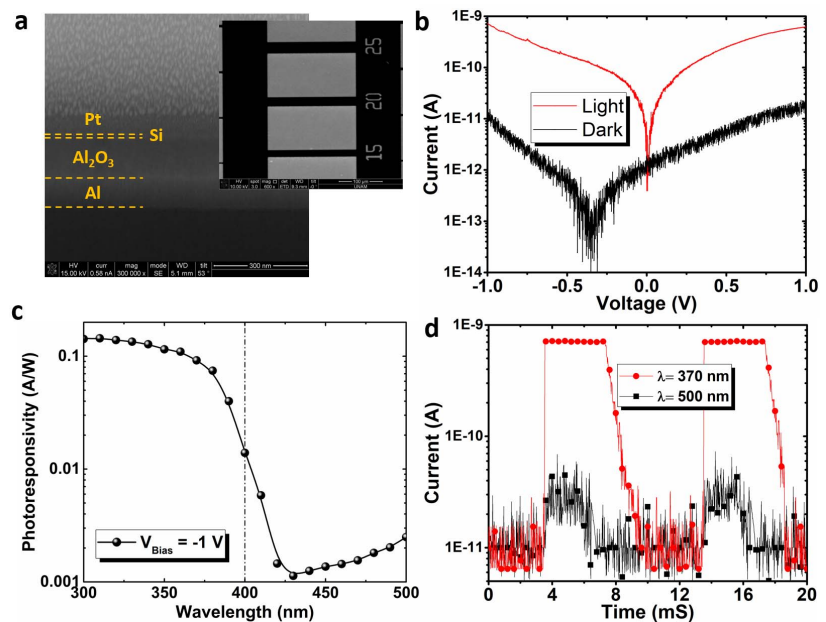

Fig. 3. (a) Cross-sectional and top SEM images of the photodetector, (b) I-V characteristic, (c) photoresponsivity, and (d) I-t response of the photodetector for different dark and light conditions.

Fig. 2(a)-(c) depict the absorbed power data inside the Si layer. To have a better qualitative comparison, the average UV $(300 \mathrm{~nm}-400 \mathrm{~nm})$ and visible $(400 \mathrm{~nm}-500 \mathrm{~nm})$ light absorptions have been calculated and exhibited in Fig. 2(d). The $1 \mathrm{~nm}$ thick case has quite negligible visible absorption while the UV absorption is high. The UV response becomes stronger while the visible light absorption is still quite small for $4 \mathrm{~nm}$ thick Si layer. This behavior is diminished in the case of $10 \mathrm{~nm}$ thick top layer.

Looking at the average absorption values, it can be understood that the optimized response belongs to $D_{S}=4 \mathrm{~nm}$, $\mathrm{D}_{\mathrm{I}}=120 \mathrm{~nm}$ where UV absorption is above 0.7 while it is just 0.02 for visible light. In order to numerically analyze the electrical response of the detector, COMSOL Multiphysics finite element method solver is utilized. For the optimal design and mentioned geometries in Fig. 2(e), the photocurrent response of the cavity in the UV region is about two order of magnitude higher compared to that of visible light, see Fig. 2(f). Inset shows data in the logarithmic scale. This response could be further explained by looking at the absorption profile of the MIS design in two different wavelengths of $370 \mathrm{~nm}$ and $500 \mathrm{~nm}$, as plotted in Fig. 2(g). While for the UV excitation $(370 \mathrm{~nm})$, most of the light is harvested in the ultrathin Si layer, this layer is transparent to visible incident light. Therefore, the formation of a symmetric metalsemiconductor-metal (MSM) junction (Au-Si-Au) at the top surface led to the formation of a visible blind UV detector. The band alignment and operation principle of this photodetector is illustrated in Fig. 2(h).

To verify our numerical results, the proposed optimal structure is fabricated and characterized. The structure is an MIS cavity made of $\mathrm{Al}(100 \mathrm{~nm})-\mathrm{Al}_{2} \mathrm{O}_{3}(120 \mathrm{~nm})-\mathrm{Si}(4 \mathrm{~nm})$. Afterward, inter-digitated $\mathrm{Au}$ electrodes are deposited on top of the cavity to from MSM junction. Fig. 3(a) shows cross sectional scanning electron microscope (SEM) image of the MIS structure and its top view showing the Au electrodes. $\mathrm{Al}$ and $\mathrm{Si}$ layers are deposited using thermal evaporation with an approximate rate of $\sim 1 \mathrm{~A}^{\circ} / \mathrm{sec} . \mathrm{Al}_{2} \mathrm{O}_{3}$ layer is formed using atomic layer deposition similar to our previous study [10].
To minimize the formation of native oxide layer between the $\mathrm{Au}$ and $\mathrm{a}-\mathrm{Si}$, both layers have been deposited in the same deposition process without breaking the chamber vacuum. Finally, standard photolithography and etching (using $\mathrm{Au}$ etchant) processes are conducted on the sample to form inter-digitated electrodes. To characterize the structure, first, the permittivity data of $\mathrm{Al}$ and $\mathrm{Al}_{2} \mathrm{O}_{3}$ layers are extracted using an elipsometry device [10]. These permittivity data are employed in our simulations. To characterize the photocurrent response of the system, IV characteristic of the structure is measured for dark and $370 \mathrm{~nm}$ illuminated light conditions. As we can see from Fig. 3(b), the photocurrent is about two order of magnitude larger compared to dark one. As seen in this panel, the dark current minimum does not locate in the $0 \mathrm{~V}$ bias. This could be due to the existence of electron trapping-de-trapping in metal-semiconductor interface which is Au-Si in our case. As previously explained, in equilibrium condition, electrons are trapped in interface defects. When a negative voltage is applied, the trapped electrons can be detrapped and contribute to the overall current [30]. To have a better qualitative comparison, the photoresponsivity of the photodetector is measured from $300 \mathrm{~nm}$ to $500 \mathrm{~nm}$ under a bias voltage of $\mathrm{V}=-1 \mathrm{~V}$. The measurement setup was similar to the one used in our previous study [31]. The cavity design exhibits a photoresponsivity of $120 \mathrm{~mA} / \mathrm{W}$ at $\lambda=350 \mathrm{~nm}$ while it drops to significantly smaller values of $2.5 \mathrm{~mA} / \mathrm{W}$ for $\lambda=500 \mathrm{~nm}$, see Fig. 3(c). Therefore, the structure shows a high responsivity ratio of about 50 for UV and visible lights. These results further confirm our simulations that this cavity acts as a high performance visible blind UV detector. The speed of this UV detector has been also investigated by analyzing its transient response under a bias voltage of $-1 \mathrm{~V}$ for two incident light wavelengths of $370 \mathrm{~nm}$ (UV) and $500 \mathrm{~nm}$ (Visible), Fig. 3(d). While the structure shows a weak visible light response, the UV activity of the MIS cavity has relatively short rise and fall times. Taking all of these results into consideration, the proposed subwavelength MIS cavity-based design has high UV detection performance. The proposed cavity has not only application in photodetectors and photovoltaics, but also it can be utilized for emission applications. It is known that reducing the size of a semiconductor nanoparticle down to its exitonic Bohr radius can intensify its emission property [32], [33]. In the other side, as demonstrated in this letter, the strong lightmatter interaction in a semiconductor based cavity occurs in nanometer scale thicknesses. Therefore, it is envisioned that introduction of a thin layer of semiconductor nanoparticles into this cavity can significantly enhance its emission property. Thus, this strategy can serve as a beacon for design of efficient, ultrathin optoelectronic devices.

\section{CONCLUSION}

In this letter, we demonstrated a visible blind UV photodetector using a MIS cavity configuration. The proposed configuration shows responsivities of $120 \mathrm{~mA} / \mathrm{W}$ and $2.5 \mathrm{~mA} / \mathrm{W}$ in the UV and visible regions, respectively. This work demonstrates the functionality of strong interference in semiconductor based cavity designs to obtain spectrally engineered, ultrathin optical devices. 


\section{REFERENCES}

[1] K. Aydin, V. E. Ferry, R. M. Briggs, and H. A. Atwater, "Broadband polarization-independent resonant light absorption using ultrathin plasmonic super absorbers," Nature Commun., vol. 2, no. 517, pp. 1-7, Nov. 2011. doi: 10.1038/ncomms1528.

[2] C. Ji, K.-T. Lee, T. Xu, J. Zhou, H. J. Park, and L. J. Guo, "Engineering light at the nanoscale: Structural color filters and broadband perfect absorbers," Adv. Opt. Mater., vol. 5, no. 20, Aug. 2017, Art. no. 1700368. doi: 10.1002/adom.201700368.

[3] S. Ogawa and M. Kimata, "Metal-insulator-metal-based plasmonic metamaterial absorbers at visible and infrared wavelengths: A review," Materials, vol. 11, no. 3, p. 458, Mar. 2018. doi: 10.3390/ma11030458.

[4] N. Liu, M. Mesch, T. Weiss, M. Hentschel, and H. Giessen, "Infrared perfect absorber and its application as plasmonic sensor," Nano Lett., vol. 10, no. 7, pp. 2342-2348, 2010.

[5] A. Tittl, P. Mai, R. Taubert, D. Dregely, N. Liu, and H. Giessen, "Palladium-based plasmonic perfect absorber in the visible wavelength range and its application to hydrogen sensing," Nano Lett., vol. 11, no. 10 , pp. 4366-4369, 2011.

[6] A. Ghobadi, H. Hajian, M. Gokbayrak, S. A. Dereshgi, A. Toprak, B. Butun, and E. Ozbay, "Visible light nearly perfect absorber: An optimum unit cell arrangement for near absolute polarization insensitivity," Opt. Express, vol. 25, no. 22, pp. 27624-27634, Oct. 2017. doi: 10.1364/OE.25.027624.

[7] Z. Li, S. Butun, and K. Aydin, "Large-area lithography-free super absorbers and color filters at visible frequencies using ultrathin metallic films," ACS Photon., vol. 2, no. 2, pp. 183-188, Feb. 2015. doi: 10.1021/ph500410u.

[8] Z. Li, E. Palacios, S. Butun, H. Kocer, and K. Aydin, "Omnidirectional, broadband light absorption using large-area, ultrathin lossy metallic film coatings," Sci. Rep., vol. 5, no. 15137, pp. 1-22, Oct. 2015. doi: 10.1038/srep15137.

[9] A. Ghobadi, H. Hajian, S. A. Dereshgi, B. Bozok, B. Butun, and E. Ozbay, "Disordered nanohole patterns in metal-insulator multilayer for ultra-broadband light absorption: Atomic layer deposition for lithography free highly repeatable large scale multilayer growth," Sci. Rep., vol. 7, no. 1, Nov. 2017, Art. no. 15079. doi: 10.1038/s41598-01715312-w.

[10] A. Ghobadi, H. Hajian, A. R. Rashed, B. Butun, and E. Ozbay, "Tuning the metal filling fraction in metal-insulator-metal ultra-broadband perfect absorbers to maximize the absorption bandwidth," Photon. Res., vol. 6, no. 3, pp. 168-176, Feb. 2018. doi: 10.1364/PRJ.6.000168.

[11] M. Chirumamilla, A. S. Roberts, F. Ding, D. Wang, P. K. Kristensen, S. I. Bozhevolnyi, and K. Pedersen, "Multilayer tungsten-alumina-based broadband light absorbers for high-temperature applications," Opt. Mater. Express, vol. 6, no. 8, pp. 2704-2714, Aug. 2016. doi: 10.1364/OME.6.002704.

[12] H. Deng, Z. Li, L. Stan, D. Rosenmann, D. Czaplewski, J. Gao, and $X$. Yang, "Broadband perfect absorber based on one ultrathin layer of refractory metal," Opt. Lett., vol. 40, no. 11, pp. 2592-2595, May 2015. doi: 10.1364/OL.40.002592.

[13] A. Ghobadi, H. Hajian, M. Gokbayrak, B. Butun, and E. Ozbay, "Bismuth-based metamaterials: From narrowband reflective color filter to extremely broadband near perfect absorber," Nanophotonics, vol. 1, no. 1, pp. 1-10, Mar. 2019. doi: 10.1515/nanoph-2018-0217.

[14] A. Ghobadi, H. Hajian, M. C. Soydan, B. Butun, and E. Ozbay, "Lithography-free planar band-pass reflective color filter using a series connection of cavities," Sci. Rep., vol. 9, no. 1, Jan. 2019, Art. no. 290. doi: 10.1038/s41598-018-36540-8.

[15] J. Park, J.-H. Kang, A. P. Vasudev, D. T. Schoen, H. Kim, E. Hasman, and M. L. Brongersma, "Omnidirectional near-unity absorption in an ultrathin planar semiconductor layer on a metal substrate," ACS Photon., vol. 1, pp. 812-821, Aug. 2014. doi: 10.1021/ph500093d.

[16] M. A. Kats, R. Blanchard, P. Genevet, and F. Capasso, "Nanometre optical coatings based on strong interference effects in highly absorbing media," Nature Mater, vol. 12, no. 1, pp. 20-24, Oct. 2012. doi: 10.1038/NMAT3443.
[17] Y. Zhang, W. Liu, Z. Li, H. Cheng, Y. Zhang, G. Jia, S. Chen, and J. Tian, "Ultrathin polarization-insensitive wide-angle broadband near-perfect absorber in the visible regime based on few-layer $\mathrm{MoS}_{2}$ films," Appl. Phys. Lett., vol. 111, no. 11, Sep. 2017, Art. no. 111109. doi: 10.1063/1.4992045.

[18] D. Jariwala, A. R. Davoyan, G. Tagliabue, M. C. Sherrott, J. Wong, and H. A. Atwater, "Near-unity absorption in van der Waals semiconductors for ultrathin optoelectronics," Nano Lett., vol. 16, no. 9, pp. 5482-5487, Aug. 2016.

[19] F. F. Schlich and R. Spolenak, "Strong interference in ultrathin semiconducting layers on a wide variety of substrate materials," Appl. Phys. Lett., vol. 103, no. 21, Nov. 2013, Art. no. 213112. doi: 10.1063/1. 4833537.

[20] S. S. Mirshafieyan, H. Guo, and J. Guo, "Zeroth order fabry-perot resonance enabled strong light absorption in ultrathin silicon films on different metals and its application for color filters," IEEE Photon. J., vol. 8, no. 5, Sep. 2016, Art. no. 6804912. doi: 10.1109/JPHOT.2016. 2609143.

[21] G. Liu, Y. Nie, G. Fu, X. Liu, Y. Liu, L. Tang, and Z. Liu, "Semiconductor meta-surface based perfect light absorber," Nanotechnology, vol. 28, no. 16, Mar. 2017, Art. no. 165202. doi: 10.1088/1361-6528/ aa6613.

[22] A. Ghobadi, H. Hajian, B. Butun, and E. Ozbay, "Strong lightmatter interaction in lithography-free planar metamaterial perfect absorbers," ACS Photon., vol. 5, no. 11, pp. 4203-4221, Oct. 2018. doi: 10.1021/acsphotonics.8b00872.

[23] X. Zhenyang, S. Haomin, K. Munho, Z. Ming, C. Tzu-Hsuan, L. Dong, Y. Xin, X. Kanglin, M. Hongyi, W. Xudong, X. Fengnian, Y. Zongfu, M. Zhenqiang, and G. Qiaoqiang, "Single-crystalline germanium nanomembrane photodetectors on foreign nanocavities," Sci. Adv., vol. 3 no. 7, pp. 1-9, Jul. 2017. doi: 10.1126/sciadv.1602783.

[24] H. Song, L. Guo, Z. Liu, K. Liu, X. Zeng, D. Ji, N. Zhang, H. Hu, S. Jiang, and Q. Gan, "Nanocavity Enhancement for ultra-thin film optical absorber," Adv. Mater, vol. 26, no. 17, pp. 2737-2743, May 2014. doi: 10.1002/adma.201305793.

[25] S. Pizzini, Advanced Silicon Materials for Photovoltaic Applications. Chichester, U.K.: Wiley, 2012.

[26] M. Casalino, U. Sassi, I. Goykhman, A. Eiden, E. Lidorikis, S. Milana, D. de Fazio, F. Tomarchio, M. Iodice, G. Coppola, and A. C. Ferrari, "Vertically illuminated, resonant cavity enhanced, graphene-silicon schottky photodetectors," ACS Nano, vol. 11, no. 11, pp. 10955-10963, Nov. 2017. doi: 10.1021/acsnano.7b04792.

[27] M. Selim and S. Strite, "Resonant cavity enhanced photonic devices," J. Appl. Phys., vol. 78, no. 2, pp. 607-639, Mar. 1995. doi: $10.1063 / 1.360322$.

[28] K. C. Balram, R. M. Audet, and D. A. B. Miller, "Nanoscale resonantcavity-enhanced germanium photodetectors with lithographically defined spectral response for improved performance at telecommunications wavelengths," Opt. Express, vol. 21, no. 8, pp. 10228-10233, Apr. 2013. doi: 10.1364/OE.21.010228.

[29] LumericalSolut Inc. Accessed: 2014. [Online]. Available: http://www.lumerical.com/tcad-products/fdtd/

[30] C.-J. Lee, C.-H. Won, J.-H. Lee, S.-H. Hahm, and H. Park, "GaN-based ultraviolet passive pixel sensor on silicon (111) substrate," Sensors, vol. 19, no. 5, p. 1051, Mar. 2019. doi: 10.3390/s19051051.

[31] T. G. Ulusoy, A. Ghobadi, and A. K. Okyay, "Surface engineered angstrom thick $\mathrm{ZnO}$-sheathed $\mathrm{TiO}_{2}$ nanowires as photoanodes for performance enhanced dye-sensitized solar cells," J. Mater. Chem. A, vol. 2, no. 40, pp. 16867-16876, Aug. 2014. doi: 10.1039/c4ta03445g.

[32] A. Ghobadi, T. Gamze, U. Ghobadi, A. K. Okyay, and E. Ozbay, "Emerging photoluminescence from defective vanadium diselenide nanosheets," Photonic Res., vol. 6, no. 4, pp. 244-253, Mar. 2018. doi: 10.1364/PRJ.6.000244.

[33] T. G. U. Ghobadi, A. Ghobadi, T. Okyay, K. Topalli, and A. K. Okyay, "Controlling luminescent silicon nanoparticle emission produced by nanosecond pulsed laser ablation: Role of interface defect states and crystallinity phase," RSC Adv., vol. 6, no. 113, pp. 112520-112526, Nov. 2016. doi: 10.1039/C6RA24412B. 